\title{
Los agrupamientos escolares a debate
}

\section{School grouping under discussion}

\author{
Maria Amparo Calatayud Salom \\ amparo.calatayud@uv.es \\ Universitat de València. España
}

\section{Resumen}

Este artículo invita a la reflexión sobre cómo los modelos de agrupamiento del alumnado influyen directamente en el éxito escolar. La literatura científica internacional desde hace décadas ha demostrado que la separación por niveles de rendimiento resulta perjudicial, sobre todo, para el alumnado que está en los grupos de más bajo nivel, ya que éste realiza menos actividades de aprendizaje instrumental, recibe menor contenido, el ritmo de aprendizaje es mucho más lento, perjudicando el rendimiento académico de todos y obteniendo peores resultados. En cambio, los resultados de investigaciones internacionales evidencian que las agrupaciones heterogéneas que, además, introducen los recursos existentes dentro del aula para dar apoyo al docente, mejoran el rendimiento académico de todo el alumnado, a la vez que mejoran su autoestima. Por tanto, los agrupamientos escolares en el sistema educativo español deberían de tomar en consideración estos andamiajes para proporcionar un aprendizaje de calidad a todos los estudiantes y no sólo a unos pocos.

Palabras clave: inclusión; agrupamientos flexibles; éxito escolar; agrupaciones heterogéneas; rendimiento académico.

\section{Abstract}

This article invites reflection on how the student grouping models directly influence school success. International scientific literature for decades has shown that separation by performance levels is detrimental especially for students in the lower-level groups, since the latter performs less instrumental learning activities, receives less content, Learning is much slower, hurting all their academic performance and getting worse results. On the other hand, the results of international research show that heterogeneous groups, which also introduce the existing resources within the classroom to support the teacher, improve the academic performance of all students, while improving their self-esteem. Therefore, school clusters in the Spanish education system should take these scaffolds into account to provide quality learning for all students and not just a few.

Keywords: inclusion; flexible grouping; school success; heterogeneous grouping; academic performance.

Recibido / Received: 15-09-2018

Aceptado / Accepted: 22-02-2018

Cómo referenciar este artículo / How to reference this article:

Calatayud, M. A. (2018). Los agrupamientos escolares a debate. Tendencias Pedagógicas, 32, 5-14. doi: $10.15366 /$ tp2018.32.001 


\section{Entresijos de los agrupamientos escolares}

“Abordar los entresijos de la organización del alumnado es fundamental puesto que condiciona no sólo los procesos de enseñanza y aprendizaje, sino también, la manera de convivir y de relacionarse entre los alumnos" (Calatayud, 2009, 12).

Los profesionales de la educación han de ser conscientes de la importancia y de cuáles son los condicionantes que determinan el agrupar a los estudiantes de una manera u otra en el aula. Indiscutiblemente, los modelos de agrupamiento del alumnado influyen directamente en el éxito escolar. Es el propio centro quien tiene que tomar la decisión sobre cómo organizar a los estudiantes, sobre cuál es la modalidad de agrupamiento más adecuada según los objetivos, contenidos, actividades, recursos, temporalización, actividades de evaluación, etc. que tiene previstas realizar durante el proceso de enseñanza y aprendizaje.

La organización del alumnado refleja y esconde un modo particular de entender la educación, la escuela, la atención a la diversidad, etc. Esas concepciones como señala Borrell (1986) son debidas a factores cualitativos como lo pueden ser: la filosofía de la educación, los objetivos propuestos, etc. que, por supuesto, lo que hacen es justificar cada solución organizativa referida al alumnado. Es decir, detrás, muchas veces, de una agrupación de alumnos u otra se esconde un enfoque de enseñanza determinado que, por supuesto, no hay que obviar. Además, la agrupación de los estudiantes también depende de aspectos relacionados con la legislación vigente, el espacio y dimensiones del centro, tipo de escuela, cantidad de discentes, las directrices marcadas en el proyecto educativo y sus implicaciones en el proyecto curricular de la etapa, etc.

Del mismo modo que es transcendente que el docente se cuestione ¿cómo organizar y distribuir los contenidos? ¿Cómo enseñar? ¿Cómo trabajar de forma globalizada o interdisciplinar las competencias? ¿Cómo y para qué evaluar? etc., es igualmente relevante ¿cómo agrupar a los estudiantes para que éstos aprendan más y mejor? Y, no sólo ello, sino que también ha de ser conocedor de que cada tipo de agrupamiento del alumnado se relaciona con aspectos afectivos y sociales, determina la manera de convivir y de relacionarse entre los estudiantes, favorece la autonomía o no, etc. De ahí, que la organización del alumnado tenga más importancia de la que en un principio pueda parecer. Diversas evaluaciones internacionales e investigaciones nacionales nos muestran efectos positivos o negativos en los resultados académicos dependiendo de las formas de agrupamiento del alumnado (Pamies y Castejón, 2015; OCDE, 2010; INCLU-ED, 2011; MIXSTRIN, 2011).

Realmente organizar a los estudiantes supone, necesariamente, crear las condiciones adecuadas para agruparlos y ubicarlos en los lugares idóneos del aula para que puedan aprender más y mejor, contar con los medios más aconsejables para propiciar la integración personal en el grupo y facilitar al máximo los procesos de enseñanza y aprendizaje posibilitando el desarrollo integral del alumno. Pero, para ello, el docente ha de conocer muy bien a sus estudiantes para saber con quién o quiénes puede "funcionar mejor" cada uno de ellos. Además, el agrupamiento ha de variar según nuestra propuesta didáctica, las necesidades, intereses y motivaciones de los alumnos de ahí, que se abogue por una flexibilidad organizativa como se explicitará en las próximas páginas.

\section{Agrupamientos escolares para el aprendizaje. Diferentes tipologías}

Como se evidencia a lo largo de este artículo, las formas de agrupamiento de los alumnos son muy diversas. Cada una de ellas encierra una forma de entender la educación distinta y, por supuesto, los aspectos curriculares quedan determinados e influenciados en mayor o menor medida por las estructuras organizativas que se crean. Como señala González, "organizar a los alumnos homogénea o heterogéneamente no remite sino a dinámicas organizativas y curriculares que promoverán la equidad educativa o, por el contrario, la segregación y el etiquetaje" (2004, p. 202).

A continuación, vamos a destacar dos tipos de clasificaciones sobre la agrupación del alumnado. Una de ellas es más tradicional y la otra, más actual, responde a las diferentes modalidades de 
agrupación del alumnado definidas por la comunidad científica internacional a partir de los resultados de la investigación INCLUD-ED (Strategies for inclusion and Social Cohesion in Europe from Education, 2006-2011), proyecto integrado del sexto Programa Marco de la Comisión Europea. Una de las investigaciones de mayor envergadura y rango científico que se han desarrollado en Europa sobre educación escolar.

\subsection{Estructuras organizativas de tipo vertical y horizontal de agrupamiento del alumnado}

\section{La organización vertical del alumnado}

La organización vertical del alumnado hace referencia a la organización por niveles educativos del sistema escolar (infantil, primaria, etc.) en los que se gradúa la enseñanza, la escolaridad y los alumnos. Básicamente, la organización vertical acoge a tres alternativas básicas: la enseñanza graduada, la enseñanza no graduada y el ciclo como unidad de organización y agrupamiento.

\section{a) La enseñanza graduada}

La graduación como marco para organizar la trayectoria escolar de los alumnos, por niveles o ciclos, es el modelo más extendido en estos momentos. La trayectoria escolar del discente se divide en diversos grados jerarquizados dotados de un currículo que el alumno ha de superar en cada uno de los ciclos, etc. Particularmente, con la enseñanza graduada aparecen términos como año académico, curso, promoción de cursos, asignatura, etc. Conceptos que como señala Gimeno (2000) "gobiernan la escolarización, y también las mentalidades de los que participan en ella (padres, alumnos y profesores)" (pág. 86). Por lo que, la escolaridad, en el modelo graduado se constituye como un modelo lineal de periodos (edades) que marca el orden del aprendizaje (González, 2003). Es un sistema de organización que suele ser utilizado porque: A) se ajusta bien a la rigidez con la que se suele organizar la escolarización de los alumnos y B) agrupa a los discentes en función de la edad lo que facilita la planificación y el control administrativo.

Por lo que las características más importantes de la enseñanza graduada, siguiendo a Antúnez y Gairín (1994), se pueden agrupar en las siguientes: a) divide el progreso de los alumnos en niveles o grados que han de ser cursados cada uno durante un año académico exactamente; b) divide cada etapa educativa en tantos niveles o grados como años escolares ha de permanecer el alumno en la misma, según la estructura del sistema educativo oficial; c) asigna a cada nivel o grado un conjunto de objetivos y contenidos de aprendizaje en forma de paquetes cerrados; d) utiliza la promoción del grupo de alumnos al nivel siguiente, de manera colectiva y simultánea; e) no suele favorecer el trabajo en colaboración del profesorado y d) no propicia estrategias metodológicas individualizadoras al no considerar el principio del progreso libre del alumnado. Además, de estas dos últimas críticas también es importante destacar que este tipo de agrupamiento somete a los discentes a numerosos controles selectivos y a una jerarquización del currículum.

b) La enseñanza no graduada

Elimina los inconvenientes de la enseñanza graduada, poniendo el énfasis en la enseñanza individualizada, al permitir que el alumno aprenda a su propio ritmo. Es un modelo poco extendido dado que requiere gran cantidad de recursos humanos, materiales, etc. En este sentido, como señala Yáñez, "la enseñanza no graduada parte del principio de que cada alumno progresa continuamente, de forma no lineal y diferente de los demás, por lo que habrá que ofertar un tipo de agrupamiento flexible y heterogéneo" (2003, p. 81). El caso más característico de enseñanza no graduada lo presentan las escuelas rurales unitarias.

\section{c) El ciclo como unidad de organización y agrupamiento}

La organización en ciclos la podemos considerar como una propuesta intermedia entre las dos anteriores, dado que posibilita un sistema más flexible para proporcionar respuestas más adecuadas a la heterogeneidad del alumnado. Antúnez y Gairín (1994) plantean que la ordenación en ciclos es 
un sistema de organización semigraduada porque presenta las siguientes características:

- Fragmenta el progreso de los alumnos en unidades de tiempo superior a un año escolar. Por lo que el alumno tiene más tiempo para alcanzar sus objetivos. El curso escolar en este sentido se desdibuja para dar paso al ciclo, unidad de aprendizaje más amplia que el curso escolar. En este sentido Bernal (2006) destaca "la importancia del concepto de ciclo como unidad temporal de organización, programación y evaluación, de tal modo que las programaciones se plantean a partir del ciclo, así como, la posible repetición de un alumno se planteará al terminar cada ciclo" (p. 209).

- Permite el progreso de los alumnos dentro del ciclo, en función de sus capacidades, mediante procesos de enseñanza que tienden a la individualización y a efectuar las adaptaciones que sean necesarias.

- Facilita una mejor organización de las actividades de recuperación y profundización.

- Ayuda a superar la idea de un docente junto a un mismo y único grupo de alumnos. Por lo tanto, los profesores pueden llegar a conocer y a responsabilizarse de todos los alumnos del ciclo.

\section{La organización horizontal del alumnado}

La organización horizontal trata de distribuir a los alumnos con características similares de escolarización (edad, años de escolarización, etc.) en grupos más pequeños para garantizar una mejor atención a las diferencias de los alumnos, por lo que este tipo de organización es el resultado de distribuir por clases a los alumnos. Los criterios que se suelen utilizar para ello son diversos y tienen que ver con el equilibrio de género, con la homogeneidad o heterogeneidad de los grupos, o con el nivel de rendimiento académico, etc. Por ello, se habla de agrupamientos homogéneos, heterogéneos y flexibles.

Los grupos homogéneos son aquellos que se establecen tomando como referente una serie de criterios ya preestablecidos como, por ejemplo: la edad, el coeficiente intelectual, etc. con el claro objetivo de que las diferencias entre los alumnos sean mínimas. El objetivo de ello, como señala Gimeno (2000) es el de "mejorar las condiciones de trabajo pedagógico, agrupando al alumno por competencias y niveles de instrucción cuyo desarrollo se considera, de alguna manera, ligado a la evolución de la edad" (p. 84).

Como han señalado investigaciones al respecto, la homogeneidad como tal no es posible dado que los alumnos difieren por su ritmo de aprendizaje, intereses, expectativas, etc. E incluso llegan a afirmar que el tipo de agrupamiento homogéneo presenta más inconvenientes que ventajas. Inconvenientes referidos, por ejemplo, a:

- Aquellos estudiantes clasificados en grupos según sus capacidades y a los que se les asigna a los grupos de "menos buenos" pueden terminar rechazando los valores académicos y sociales del centro.

- Agrupar a los alumnos por capacidades está demostrado que no produce per se, un efecto significativo en el rendimiento.

Por lo tanto, a raíz de todos estos planteamientos se aboga más por agrupamientos heterogéneos y flexibles. La heterogeneidad utilizando criterios como el orden alfabético de los apellidos, el número de orden de matriculación, etc. en la composición de los grupos de alumnos constituye, por lo tanto, una exigencia básica de democratización y equidad educativa (González, 2004).

El agrupamiento flexible, como su nombre indica, trata de agrupar a los alumnos según momentos, necesidades, propuestas metodológicas, etc. Agrupamiento que puede ser horizontal (agrupando a alumnos de diferentes niveles) o bien vertical (agrupando a alumnos de diferentes ciclos, etc.) y que es necesario que se aborde colegiadamente en el centro, dado que se tienen que delimitar muy bien los objetivos que se quieren perseguir, los recursos, la coordinación entre el 
profesorado, etc. (Santos Guerra, 1994); modalidad que trataremos con mayor profundidad más adelante.

2.2. Diferentes modalidades de agrupación del alumnado definidas por la comunidad cientifica internacional, a partir de los resultados de la investigación "INCLUD-ED" (2009-2011) y su relación con el éxito escolar: Mixture, Streaming e Inclusión.

\section{Mixture: Un único docente ante un alumnado beterogéneo}

"Mixture" hace referencia a la organización del aula tradicional, en la que se incluye a todo el alumnado de un grupo, diverso en cuanto a nivel educativo, con un único profesor. Hoy por hoy, en la sociedad en la que vivimos con una serie de fenómenos como la globalización, la inmigración, la pérdida de valores, las nuevas formas familiares, etc. (Calatayud, 2008) hace que un único profesor en el aula, resulte bastante complicado atender a la diversidad socio-cultural y a diferentes niveles de rendimiento. Por lo que en la práctica este modelo plantea serias dificultades derivadas del contexto socio-educativo actual. Como señala la profesora Valls (2012) el docente, para satisfacer las demandas de esta diversidad, necesita más apoyos dentro y fuera del aula, por lo que se hace necesario implicar a toda la comunidad en el aprendizaje y gestión de los centros educativos.

\section{Streaming: separación del alumnado por niveles de rendimiento}

"Streaming" ha sido definida por la Comisión Europea (2006) como la adaptación del currículum a distintos grupos de alumnos, de acuerdo con su rendimiento, dentro de un mismo centro escolar. Como señala Morán, Molina y Siles (2012) la forma más común de streaming consiste en separar al alumnado por niveles de rendimiento en diferentes aulas o dentro de la misma aula, en todas o algunas asignaturas, pero añadiendo más recursos humanos para atender a los diferentes grupos de alumnos (Parsons y Hallam, 2015).

El streaming suele ser una práctica bastante común en Europa. Por ejemplo, en Finlandia que, como se sabe está a la cabeza de Europa en los resultados de las evaluaciones internacionales como PISA, prohibió en los años 80 en su legislación este tipo de agrupamiento. Watanabe (2008), señala que la separación del alumnado por niveles limita el efecto positivo sobre la mejora del aprendizaje que tienen las interacciones entre el alumnado de rendimiento alto y el alumnado de rendimiento medio y bajo.

Desde el proyecto INCLUD-ED se ha propuesto una clasificación de las diversas prácticas de streaming, así como, los criterios para facilitar su análisis. A continuación, se van a proponer dichas modalidades para que luego podamos compararlas con las modalidades de inclusión que es la tendencia internacional hacia la cual deberíamos dirigirnos para tratar de alcanzar el éxito educativo para todos los estudiantes, pero desde la heterogeneidad en el aula. En la siguiente tabla planteada por Morán, Molina y Siles (2012, p. 15) mostramos las 4 diferentes formas de streaming y sus características principales:

1. Organización de las actividades de aprendizaje por niveles de rendimiento.

- La enseñanza se adapta a las diferentes necesidades y ritmos de aprendizaje del alumnado.

- El alumnado con niveles de rendimiento alto y bajo se agrupan por separado, en la misma aula o en clases separadas.

- El agrupamiento por niveles de rendimiento normalmente se emplea para la enseñanza de materias instrumentales. Aparece más frecuentemente en educación secundaria y suele conducir a distintos itinerarios educativos.

- Esta forma de agrupamiento puede incidir negativamente en el éxito escolar y en la integración social.

- La asignación de alumnado a distintos grupos suele basarse en la decisión individual del profesorado del centro escolar.

- El alumnado perteneciente a colectivos vulnerables se ubica con mayor frecuencia en los 
grupos de nivel bajo.

2. Grupos de refuerzo o de apoyo separados del grupo de referencia.

- Se crean para niños con necesidades educativas especiales o para aquellos que se encuentran en riesgo de exclusión social.

- Estos alumnos se separan de su grupo de referencia durante el horario escolar para recibir apoyo educativo.

- Esta medida se suele adoptar con alumnado que requiere educación especial, así como, con inmigrantes, miembros de minoría culturales y aquellos que no dominan la lengua de instrucción.

- Una de sus consecuencias es el etiquetaje de alumnos y una disminución del nivel de la instrucción recibida.

3. Adaptaciones curriculares individuales "exclusoras"

- Se adapta el currículo oficial al nivel de aprendizaje de un alumno en concreto (o de un grupo de alumnos) reduciendo el nivel de dificultad de los contenidos.

- Esta medida suele llevarse a cabo con grupos particulares de alumnado: alumnos de educación especial, inmigrantes y quienes han de aprender la lengua de instrucción.

4. Optatividad "Exclusora"

- La elección de una materia o un conjunto de materias desemboca en desigualdad de oportunidades académicas y sociales en el futuro.

- Cuando esto sucede, la elección de materias o itinerarios suele estar fuertemente asociada al estatus socioeconómico familiar y a las expectativas del profesorado.

3. Tendencias de agrupamientos escolares para el éxito escolar: la inclusión y el agrupamiento flexible

\subsection{Inclusión: heterogeneidad en el aula y éxito educativo para todos}

La inclusión ha sido definida por la comunidad científica internacional como aquellas prácticas educativas que logran una respuesta satisfactoria a la diversidad de alumnado, tanto en sus niveles de aprendizaje como en sus contextos socioeducativos, contribuyendo, por tanto, a la igualdad no sólo de oportunidades sino también de resultados y al éxito de todos y todas, pero manteniendo la heterogeneidad de las aulas, sin separar por niveles de rendimiento. Como señala Morán, Molina y Siles (2012), frente a la diversidad en las aulas, en lugar de adaptar el contenido al nivel del alumnado, creando grupos de nivel para ello, las iniciativas que se incluyen dentro de esta modalidad se basan en mantener la heterogeneidad del aula pero incorporando recursos en su interior, proporcionando el apoyo necesario en un entorno compartido y con el mismo contenido de aprendizaje. Por lo tanto, como describe Duque et al. (2012) el objetivo de esta modalidad es conseguir que el curriculum ordinario sea accesible a todo el alumnado, sin reducirlo, entrando otras personas adultas en el aula (profesorado de apoyo, familiares, voluntariado, etc.) a la vez que se promueve la ayuda entre el alumnado para que así todos asimilen los contenidos de aprendizaje. A continuación, se presentan las diferentes modalidades de inclusión que cada vez más se están potenciando en las aulas para tratar de conseguir el éxito educativo para todos desde la heterogeneidad en el aula (Duque et al., 2012, p. 21).

1. Grupos heterogéneos con una reorganización de recursos humanos.

- Este tipo de inclusión consiste en proporcionar más apoyo mediante la redistribución de recursos humanos en aulas con alumnado diverso. En la mayoría de los casos es el profesorado quien proporciona este apoyo, aunque también pueden intervenir en el aula familiares o miembros de la comunidad.

- Los grupos interactivos han resultado ser una forma muy útil de agrupamiento heterogéneo con una distribución alternativa de recursos. En los grupos interactivos, las clases se organizan en pequeños grupos heterogéneos de alumnos, cada uno a cargo de una persona adulta (profesor, voluntarios, etc.) que facilita la interacción y la colaboración entre el alumnado. El tutor se encarga, de coordinar las actividades en el aula y de proporcionar apoyo cuando es preciso. 
- En la mayoría de los centros, los recursos humanos adicionales se destinan a grupos específicos de alumnos, como, por ejemplo, a los que se considera que requieren educación especial, inmigrantes, minorías étnicas, etc. Este apoyo, permite, por tanto, a este tipo de alumnado permanecer dentro de su grupo de referencia.

2. Desdobles en grupos heterogéneos.

- Distintos profesores se hacen cargo de diferentes grupos heterogéneos de alumnos. El grupo de referencia se puede desdoblar, por ejemplo, en dos subgrupos de 12 niños o niñas, sin hacer una selección por niveles de aprendizaje.

- Este tipo de agrupamiento es frecuente en algunas materias (en lengua o matemáticas) y permite organizar la clase de manera distinta y reducir la ratio profesor/alumno.

3. Ampliación del tiempo de aprendizaje.

- La posibilidad de ampliar el tiempo de aprendizaje o de organizar actividades académicas adicionales es más común en el caso del alumnado que vive en áreas socialmente deprimidas o pertenece a minorías.

- Esto puede traducirse en una ampliación del horario escolar, en proporcionar ayuda para el alumnado y sus familias mediante clases en el centro o en casa, y en la oferta de actividades educativas en periodos vacacionales y en horario extraescolar durante el curso. Por ejemplo, el Ministerio de Educación ofrece apoyo a los centros educativos en relación a la ampliación del tiempo de aprendizaje mediante el Plan PROA (Programa de Refuerzo, Orientación y Apoyo).

4. Adaptaciones curriculares individuales inclusivas.

- La adaptación curricular inclusiva no se concibe como una reducción de los contenidos que un alumno ha de adquirir, sino como una adaptación de los métodos de enseñanza para facilitar al alumno el aprendizaje.

5. Optatividad inclusiva.

- La elección de materias optativas no se basa en el nivel de rendimiento del alumnado, sino en sus preferencias y no conduce a un callejón sin salida.

- No reduce las oportunidades educativas y sociales del alumnado en el futuro, sino que garantiza que éste tendrá las mismas posibilidades en cualquier opción que escoja.

\subsection{El agrupamiento flexible: una modalidad facilitadora del éxito escolar.}

Es de destacar que en nuestro país ha habido interesantes experiencias relacionadas con agrupar a los alumnos con criterios diferentes a la edad cronológica. Por ejemplo, los planteamientos multinivel de agrupamientos flexibles, las propuestas didácticas que intentan personalizar el aprendizaje a través de una organización curricular, agrupando al alumnado en talleres, proyectos o por intereses en las escuelas de Educación Primaria. Indudablemente, como se puede apreciar estas experiencias tienen en común que consideran los procesos de aprendizaje de los alumnos por encima de los procesos de enseñanza de los profesores. De ahí, que se abogue por defender la flexibilidad organizativa dado que ello supone combinar diferentes agrupamientos en el centro y dentro del aula, en función siempre de las características, intereses, motivaciones y necesidades de aprendizaje de los estudiantes. Por supuesto, optar por el agrupamiento flexible conlleva innumerables ventajas, por ejemplo, algunas de ellas son las siguientes:

- Impulsa el aprendizaje cooperativo en el aula, según el cual los alumnos aprenden unos de otros y en el que el éxito de cada uno depende de que el conjunto de sus compañeros alcance las metas fijadas. La interacción entre iguales es una de las formas de aprender significativamente, que facilita el que el aula se considere como una comunidad de aprendizaje. Por tanto, estimula la discusión y la necesidad de explicitar ante los compañeros y compañeras su propio pensamiento propiciando el que el alumno construya el conocimiento, lo que le motiva para que aprenda a aprender.

- Crea un clima de trabajo orientado a metas definidas en común, abierto al intercambio y a la discusión de las ideas amparadas siempre en la fuerza de los argumentos, evitando la imposición de puntos de vista bajo criterios de superioridad. Por lo que fomenta la elaboración y maduración de las ideas de los alumnos de modo que generan un pensamiento 
crítico y creativo, propio y fundamentado.

- Estimula la resolución de problemas, la creatividad y la utilización de la investigación como método para que el alumno a través del agrupamiento flexible pueda convertirse en el protagonista del proceso de enseñanza y aprendizaje.

Las formas de organización mediante grupos flexibles son muy variadas. Antúnez y Gairín (1994, 120-122) destacan como modalidades que se ponen en práctica en los centros escolares, con bastante frecuencia, las siguientes:

- El sistema multiniveles, que consiste en organizar el currículo de algunas áreas o materias mediante una secuenciación de los contenidos muy pormenorizada. Cada alumno puede progresar en el itinerario que se le ofrece según su ritmo y capacidades, de forma que los alumnos que pertenecen a un mismo grupo-clase pueden estar trabajando contenidos curriculares de niveles de dificultad y profundización diferentes durante un mismo periodo de tiempo.

- La organización del curriculo por proyectos, que supone un intento deliberado, estructurado y planificado de estudiar conceptos, fenómenos o problemas desde una orientación multidisciplinar, tratando de construir respuestas que posibiliten el trabajo cooperativo. Por lo tanto, plantean una temática para trabajar de manera sistemática y organizada, significativa para el alumno y relevante para aprender los aspectos que conforman la cultura. Enmarcan y dan coherencia a las situaciones educativas escolares, ya que analizan los aspectos relevantes a la cultura que han de aprender los estudiantes, organizando a su alrededor la actividad escolar y el contenido del aprendizaje.

- Los rincones o áreas de actividades y los talleres, que permiten organizar partes del currículo mediante agrupamientos flexibles que se constituyen en torno a núcleos de trabajo dispuestos ordenadamente para ayudar a desarrollar unas determinadas capacidades. Las áreas de actividad están cuidadosamente diseñadas en función de los intereses y capacidades de los alumnos y permiten, por tanto, el trabajo cooperativo.

Además de estas modalidades organizativas, los centros de interés de Decroly y la clase cooperativa siguiendo las directrices de las técnicas de Freinet, son también otras formas de organización flexible.

- Los agrupamientos flexibles según situaciones de aprendizaje: Gran grupo, grupo medio, pequeño grupo y trabajo individual.

\section{Consideraciones finales}

Tras la lectura de este artículo, se ha visto cómo los modelos de agrupamiento del alumnado influyen directamente en el éxito escolar, tal y como han demostrado las numerosas investigaciones tanto de índole nacional como internacional. A través de los resultados de la investigación INCLUD-ED y a partir de proyecto de investigación I+D MIXSTRIN (2008-2011) en el que se realizó un estudio para conocer la implementación de las tres modalidades (mixture, streaming e inclusión) en los centros educativos públicos de primaria y secundaria en España y su relación con los resultados académicos se han extraído las siguientes conclusiones que deberían hacernos reflexionar sobre la situación actual.

Uno de los resultados más llamativos ha sido que las modalidades de streaming y mixture son las que en mayor medida se están aplicando en España y, escasamente se están trabajando las prácticas de modalidad de inclusión a través de las experiencias de centros convertidos en Comunidades de Aprendizaje.

Además, se ha evidenciado claramente que la organización tradicional del aula con un único profesor ante un alumno diverso, hoy por hoy, no puede funcionar. $\mathrm{Y}$ es, como todos sabemos, lo que en estos momentos predomina en los centros de Educación Infantil y Primaria en España. 
Asimismo, la literatura científica internacional desde hace décadas ha demostrado que la separación por niveles de rendimiento resulta perjudicial, sobre todo, para el alumnado que está en los grupos de más bajo nivel, ya que éste realiza menos actividades de aprendizaje instrumental, recibe menor contenido, el ritmo de aprendizaje es mucho más lento, perjudicando a todos su rendimiento académico y obteniendo peores resultados. Y por supuesto, además de no contribuir a una mejora de la convivencia, ni a la integración social.

En cambio, los resultados de investigaciones internacionales evidencian que las agrupaciones heterogéneas que, además, introducen los recursos existentes dentro del aula para dar apoyo al docente, mejoran el rendimiento académico de todo el alumnado, a la vez que mejoran la autoestima del estudiante y la convivencia de todo el centro escolar, sin que haya una disminución en el currículum propuesto. (Acedo, 2008; Blanco, 2008, Florian, 2013; Parsons y Hallan, 2014; Susinos y Rodríguez, 2011).

Pues bien, mi pregunta al respecto es ¿por qué los políticos y los dirigentes de un país en materia de educación no se leen estas investigaciones antes de realizar las reformas educativas? Dirigir nuestra mirada hacia el futuro necesita de andamiajes sólidos que, entre otros argumentos, sólo nos lo pueden aportar las investigaciones educativas. Pues bien, hagámosles caso.

\section{Referencias}

Acedo, C. (2008). Educación inclusiva: superando los límites. Perspectivas. Revista Trimestral de Educación Comparada, 38(1), pp. 5-16.

Antúnez, S., \& Gairín, J. (1994). La organización académica del alumnado en la organización escolar. Prácticay fundamentos. Barcelona: Graó.

Bernal, J.L. (2006). Comprender nuestros centros educativos. Zaragoza: Editores Mira.

Blanco, R. (2008). Construyendo las bases de la inclusión y la calidad de la educación en la primera infancia. Revista de Educación, 347, 33-54.

Borrell, N. (1986). Agrupamiento de alumnos. Educar, 6, pp. 145- 158.

Calatayud, M.A. (2008). La escuela del futuro. Hacia nuevos escenarios. Madrid: CCS.

Calatayud, M.A. (2009). Entresijos de los centros escolares. Desvelarlos para mejorarlos. Málaga: Aljibe.

Comisión Europea. (2006). Eficiencia y equidad en los sistemas europeos de educación y formación. Bruselas.

Duque, E., Ruíz, L., Girbés, S., \& Padrós, M. (2012). Inclusión y éxito educativo: prácticas de aprendizaje y participación de las familias en los centros educativos. Revista de Organización y Gestión Educativa, 2, pp. 19-22.

Florian, L (2013). La educación especial en la era de la inclusión: ¿El fin de la educación especial o un nuevo comienzo? Revista Latinoamericana de Inclusión Educativa, 7(2), pp. 27-36.

Gimeno, J. (2000). La educación obligatoria: su sentido educativo y social. Madrid: Morata.

González, M.T. (2004). Organización y estructuras para el trabajo y aprendizaje del profesorado y el alumnado. En Moreno Olmedilla, J.M. (Coord.), Organización y gestión de centros educativos (pp. 185206). Madrid: UNED.

González, M.T. (2003). Estructuras para el trabajo de los alumnos: los agrupamientos. En González, M.T. (Coord.), Organización y Gestión de Centros Escolares (pp. 91-105). Madrid: Prentice.

INCLUD-ED. (2006-2011). Proyecto Integrado INCLUD-ED "Strategies for inclusionn and social cohesion in Europe education" (2006-2011). Recuperado de: http:// www.ub.edu/included/es/about.htm.

MIXSTRIN, (2008-2011). Plan Nacional I+D+i. Formas de agrupación del alumnado y su relación con el éxito escolar. Recuperado de: http://mixstrin.wordpress.com.

Morán de Castro, C., Molina, S. y Siles, G. (2012). Aportaciones científicas a las formas de agrupación del alumnado. Revista de Organización y Gestión Educativa, 2, pp. 13-18.

OECD. (2010). PISA (2009). Results: What makes a school successful? Resources, policies and practices. Volume IV. París: OECD.

Pamies, J., \& Castejón, J. (2015). Distribuyendo oportunidades. El impacto de los agrupamientos escolares en la experiencia de los estudiantes. Revista de la Asociación de Sociología de la Educación, 8(3), pp. 335-348. 
Parsons, S., \& Hallam, S. (2014). The impact of streaming on attainment at age seven: evidence from the Millennium Cohort Study. Oxford Review of Education, 40(5), pp. 567-589. doi: 10.1080/03054985.2014.959911.

Susinos, T., \& Rodríguez, C. (2011). La educación inclusiva hoy. Reconocer al otro y crear comunidad a través del diálogo y la participación. Revista Interuniversitaria de Formación del Profesorado, 70(1), pp. 15-30.

Santos, M.A. (1994). Entre bastidores. El Lado oculto de la organización escolar. Málaga: Aljibe.

Valls, R. (2012). La separación por niveles no logra la mejora académica. Revista de Organización y Gestión Educativa, 2, pp. 35-38.

Watanabe, M. (2008). Tracking in the Era of High Stakes State Accountability Reform. Case Studies of Classroom Instruction in North Carolina Teachers. College Record, 110, pp. 489-534.

Yañez, J. (2003). Dirección de centros educativos. Madrid: Síntesis. 